\title{
Effects of varying magnetosheath flow and dissipation on the two-dimensional reconnection structure at the magnetopause
}

\author{
Lars G. Westerberg, ${ }^{\text {a) }}$ Hans O. Åkerstedt, and J. Henric Taavola \\ Division of Fluid Mechanics, Luleå University of Technology, SE-971 87 Luleå, Sweden
}

(Received 16 January 2009; accepted 21 April 2009; published online 18 May 2009)

\begin{abstract}
The stationary Riemann problem including dissipation in form of resistivity and viscosity for the reconnection structure at the dayside magnetopause is considered. Including tangential velocity shear and gradients across the complete reconnection structure from the magnetosheath side into the magnetosphere, it is shown how dissipation affects the thickness of the intermediate shock wave in the vicinity of a reconnection site. We present how plasma flow structure undergoes a significant change for a nonzero uniform plasma velocity and velocity gradient. It is also shown how the reconnection rate changes and leads to a cutoff when the velocity becomes Alfvénic. () 2009 American Institute of Physics. [DOI: 10.1063/1.3133186]
\end{abstract}

\section{INTRODUCTION}

Being a key mechanism treating the conversion of magnetic energy into kinetic energy, magnetic reconnection has, during the last 50 years, been considered to play a vital role in space-, astro-, and stellar physics as well as in laboratory plasma applications. The reconnection process dominates the transport of solar wind plasma into the terrestrial magnetosphere. Nowadays reconnection research accounts progress from both analytical, computational, and observational approaches. Having a first class laboratory for reconnection research in the form of the terrestrial magnetosphere, the joint-and highly successful-ESA/NASA Cluster II mission has come to play a significant role in the understanding of this process. In terms of reconnection at the dayside magnetopause, which is the actual situation for the present study, a number of studies have used the full potential of the Cluster II armada. ${ }^{1-4}$

With the help of constantly developed numerical codes and computer performance, a deeper view into the mechanisms which determine the reconnection process is possible to achieve. In order to investigate the energy transport coupled to magnetic reconnection and solar wind conditions, global MagnetoHydroDynamic (MHD) simulations have successfully been used. ${ }^{5-7}$ Also, in order to penetrate more into the effects of fast magnetic reconnection regimes, $\mathrm{Ugai}^{8-10}$ recently used two-dimensional (2D) and threedimensional (3D) MHD simulations.

With the objective of resolving the time evolution of the reconnection structure and investigating the development of the MHD discontinuities, a lot of work has been done during the years treating one-dimensional time-dependent simulations solving the MHD Riemann problem. ${ }^{11-16}$ In these simulations reconnection starts from an initial current sheet separating antiparallel magnetic fields together with an imposed normal component of the magnetic field. Here the tangential velocity shear is also included to simulate plasma flows in the flank and high-latitude magnetopause. In the ideal MHD

\footnotetext{
${ }^{a)}$ Electronic mail: lgwe@1tu.se.
}

limit a set of MHD discontinuities develop. Including finite resistivity, the simulations show that resistivity does not change the jumps of physical quantities across the discontinuities, except the rotational discontinuity which becomes time dependent and is called time-dependent intermediate shock (TDIS). The thickness of the TDIS waves increases as the square root of time, which is typical for the case of waves with dissipation but with no nonlinear wave steepening effect. It is therefore interesting to consider the stationary counterpart of this structure for similar magnetic reconnection in 2D and investigate if the thickness of the rotational discontinuity increases away from the reconnection site. In a series of papers of 2D and 3D steady reconnections, ${ }^{17-20}$ it has been shown that this is, in fact, the case for the rotational discontinuity. Westerberg and Åkerstedt ${ }^{20}$ showed that data from the Cluster spacecraft armada support this view. In the present paper we therefore consider the stationary Riemann problem including dissipation in the form of resistivity and viscosity, and we explicitly also show how dissipation affects the thickness of this wave. We also include the tangential velocity shear and gradients across the complete reconnection structure from the magnetosheath side into the magnetosphere.

We will not consider the important matching between the outflow region about the reconnection site and the diffusion region. A successful asymptotic matching of this kind has quite recently been performed by Erkaev et al. ${ }^{21}$ for twodimensional symmetric reconnection. Due to its complexity this analysis is not considered in the present paper. Also, since the purpose of the present work is the implications of reconnection on the outflow surrounding plasma, we just pragmatically suppose that such kind of matching to the diffusion region exists. Since we consider the Petschek reconnection case in this paper, it is reasonable that the results of the analysis of the diffusion region and the matching to the outer flow given by Erkaev et al. ${ }^{21}$ are applicable also to the present case, i.e., with a diffusion region of small size. The analysis by Erkaev et al. ${ }^{21}$ of the 2D Petschek reconnection mechanism clearly shows that the mechanism works only if there is a large anomalous resistivity localized to the diffu- 
sion region and that the background resistivity of the region outside the diffusion region is much smaller. In the present study we consider the outflow region and especially the structure of the intermediate shock. This means that we should have a resistivity much smaller than the resistivity inside the diffusion region. Whether this background resistivity is of Spitzer type or some anomalous resistivity is not known without measurements. However, as shown from Cluster data, ${ }^{20}$ the measured thickness of the intermediate shock layer cannot be explained by Spitzer resistivity and therefore the resistivity has to be of anomalous type. To cope with the Petschek mechanism of reconnection, we therefore assume a large anomalous resistivity inside the diffusion region and a smaller background anomalous resistivity in the outflow region. The diffusion region also imposes the initial structure and thickness of the intermediate shock.

The inclusion of a shear magnetic field component $\left(B_{y}\right)$ has not been considered in the present 2D approach. However, it is of great interest to develop the present study to cover a full 3D analysis. Preliminary results for the outer solutions indicate that the inclusion of $B_{y}$ does not affect the flow dynamics significantly. The solutions obtained for respective coordinates are linear combinations of the present $(x, z)$ coordinates, indicating the flow in a frame of reference which is rotated along the present $z$-axis. The full analysis of the flow through the transition of the intermediate shock wave-and the corresponding development of the intermediate shock wave-is very extensive including an analysis of the jump conditions across the magnetopause shock structure. This is a highly interesting development of the present paper, but is not considered at the actual stage in the analysis.

The outline of the present paper is as follows. In Sec. II we present the governing equations and method of solution for the magnetosheath flow. With Sec. III the approximate solutions for the outer flow is complete and ready to be coupled with the $2 \mathrm{D}$ reconnection model. This is done in Sec. IV, while in Sec. V the solutions for the outer flow are matched with the solutions in the transition Alfvén layer. In Sec. VI the results are viewed and discussed. The study is wrapped up and summarized in Sec. VII.

\section{MAGNETOSHEATH FLOW MODEL}

In this section we solve the ideal MHD equations governing the plasma flow in the magnetosheath. This is done by expanding the magnetic field and plasma velocity along uniform fields.

We consider a curvilinear coordinate system where $x$ points in the normal direction to the magnetopause surface, and $z$ in the tangential direction. See Fig. 1.

In an ideal incompressible MHD treatment, the reconnection structure consists of discontinuities corresponding to an Alfvén wave $\mathrm{A}$, a contact discontinuity $\mathrm{C}$, and a slow shock S, see Fig. 2. These discontinuities are resolved by including nonideal effects such as resistivity and viscosity. While the resolved contact and shock waves approach a finite thickness due to a balance between nonlinearity and dissipation, the Alfvén wave thickness grows away from the reconnection site. This behavior of the Alfvén wave is due to

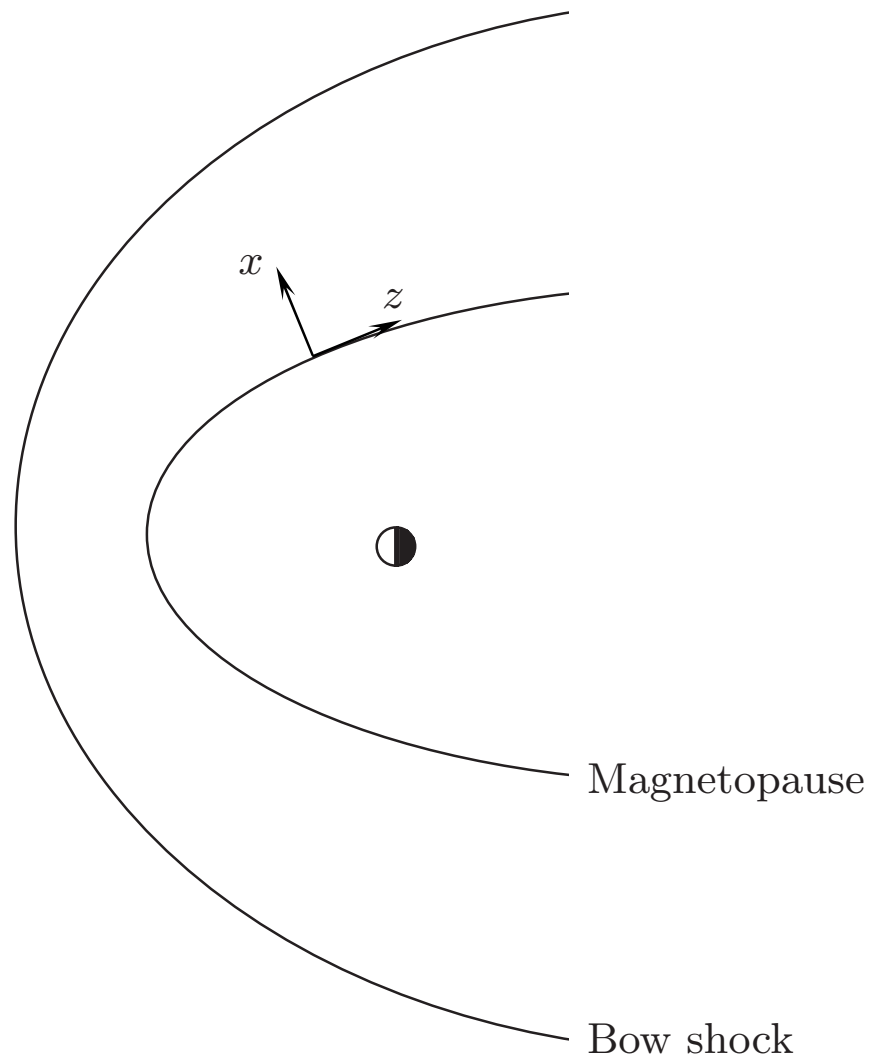

FIG. 1. The curvilinear coordinate system. The conventional coordinate system in space physics is used, i.e., $x$ pointing normal to the magnetopause surface and the $z$-component lying in the tangential direction of the magnetopause.

the lack of nonlinear terms in the equations governing the evolution of this wave. Effects of dissipation on the Alfvén layer are therefore of much more importance than the corresponding effects on the contact and slow shock waves. In this paper we therefore treat the contact wave and the slow shocks as discontinuities while including nonideal effects in the description of the Alfvén wave.

\section{A. Governing equations}

We consider the ideal Ohm's law and the ideal MHD equation of motion on dimensionless form,

$$
\mathbf{E}+\mathbf{u} \times \mathbf{B}=0,
$$

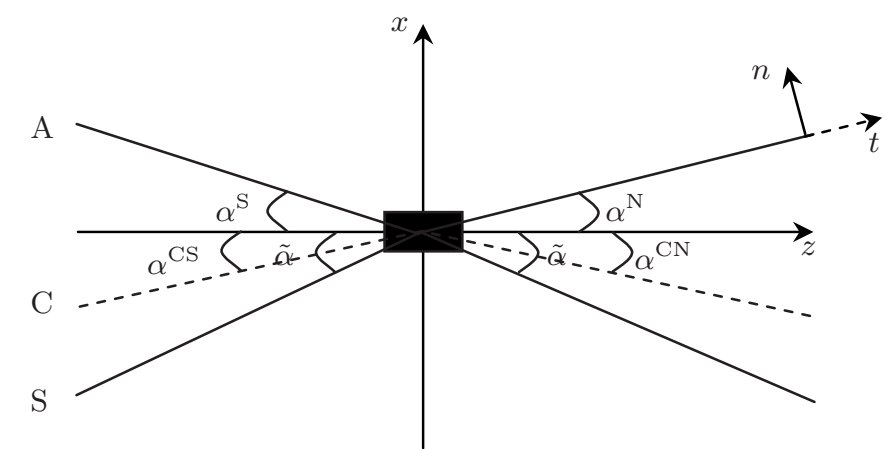

FIG. 2. Evolution of the incompressible shock waves north and south of the reconnection site. A is the Alfvén wave, $\mathrm{S}$ is the incompressible limit of a slow shock, and $\mathrm{C}$ is the contact discontinuity. See the Appendix for analytical expressions for respective angle. 


$$
(\mathbf{u} \cdot \nabla) \mathbf{u}=-\nabla p+(\mathbf{B} \cdot \nabla) \mathbf{B} .
$$

For an approximate solution the magnetic field and velocity are expanded in terms of the small electric field $E$ about uniform fields $U_{0} \hat{\mathbf{z}}$ and $B_{0} \hat{\mathbf{z}}$, such that

$$
\begin{aligned}
& \mathbf{u}=\mathbf{u}_{0}+\mathbf{u}_{1}+\mathbf{u}_{2}+\cdots, \\
& \mathbf{B}=\mathbf{B}_{0}+\mathbf{B}_{1}+\mathbf{B}_{2}+\cdots,
\end{aligned}
$$

where the leading order terms are considered to be of the order $O\left(E^{0}\right)$. Following terms scales as $O\left(E^{1 / 2}\right)$ and $O\left(E^{1}\right)$, respectively. In order to include a velocity variation with $z$, we assume this to be of order $O\left(E^{1 / 2}\right)$.

\section{B. Solutions to leading order $O\left(E^{0}\right)$}

Applying Eq. (3) in Eq. (1) gives to leading order

$$
\begin{aligned}
& \mathbf{u}_{0}=U_{0} \hat{\mathbf{z}}, \\
& \mathbf{B}_{0}=B_{0} \hat{\mathbf{z}},
\end{aligned}
$$

which satisfies

$$
\begin{aligned}
& \nabla \cdot \mathbf{u}_{0}=0, \\
& \nabla \cdot \mathbf{B}_{0}=0 .
\end{aligned}
$$

\section{Solutions to order $O\left(E^{1 / 2}\right)$}

To order $O\left(E^{1 / 2}\right)(1)$ results in

$$
\mathbf{u}_{0} \times \mathbf{B}_{1}+\mathbf{u}_{1} \times \mathbf{B}_{0},
$$

which together with Eq. (5) gives

$$
B_{1 x}=\frac{B_{0}}{U_{0}} u_{1 x} .
$$

Assuming

$$
u_{1 z}=Q z,
$$

where $Q$ is the velocity gradient, Eq. (11) together with the condition

$$
\nabla \cdot \mathbf{u}_{1}=0
$$

results in

$$
u_{1 x}=-Q x .
$$

Equation (13) in Eq. (10) then gives

$$
B_{1 x}=-\frac{B_{0}}{U_{0}} Q x .
$$

The divergence free property of $\mathbf{B}_{1}$ gives

$$
B_{1 z}=\frac{B_{0} Q}{U_{0}} z
$$

For the pressure we obtain

$$
\hat{\mathbf{x}}: \quad \frac{\partial p_{1}}{\partial x}=0,
$$

$$
\hat{\mathbf{z}}: \quad \frac{\partial p_{1}}{\partial z}=\left\{\left(\frac{B_{0}}{U_{0}}\right)^{2}-1\right\} U_{0} Q .
$$

In summary, we have at the present stage to the order $O\left(E^{1 / 2}\right)$

$$
\begin{aligned}
& u_{1 x}=-Q x, \\
& u_{1 z}=Q z, \\
& B_{1 x}=-\frac{B_{0}}{U_{0}} Q x, \\
& B_{1 z}=\frac{B_{0}}{U_{0}} Q z, \\
& p_{1}=\left\{\left(\frac{B_{0}}{U_{0}}\right)^{2}-1\right\} U_{0} Q z .
\end{aligned}
$$

\section{SOLUTIONS TO ORDER O(E)}

Having obtained the solutions for up to the ordering $O\left(E^{1 / 2}\right)$, we proceed by seeking the solutions for $\mathbf{u}_{2}, \mathbf{B}_{2}$, and $p_{2}$. Equation (1) gives to order $O(E)$,

$$
\mathbf{E}+\mathbf{u}_{2} \times \mathbf{B}_{0}+\mathbf{u}_{0} \times \mathbf{B}_{2}+\mathbf{u}_{1} \times \mathbf{B}_{1} .
$$

Taking $\mathbf{B}_{0} \times(23)$ and solving for $\mathbf{u}_{2}$ results in

$$
\begin{aligned}
\mathbf{u}_{2}= & \frac{\mathbf{E} \times \mathbf{B}_{0}}{B_{0}^{2}}+\frac{1}{B_{0}^{2}}\left(\mathbf{B}_{0} \cdot \mathbf{u}_{2}\right) \mathbf{B}_{0}+\frac{1}{B_{0}^{2}}\left(\mathbf{B}_{0} \cdot \mathbf{u}_{1}\right) \mathbf{B}_{2} \\
& -\frac{1}{B_{0}^{2}}\left(\mathbf{B}_{0} \cdot \mathbf{B}_{2}\right) \mathbf{u}_{0}-\frac{1}{B_{0}^{2}}\left(\mathbf{B}_{0} \cdot \mathbf{B}_{1}\right) \mathbf{u}_{1}+\frac{1}{B_{0}^{2}}\left(\mathbf{B}_{0} \cdot \mathbf{u}_{1}\right) \mathbf{B}_{1}=0 .
\end{aligned}
$$

$\nabla \cdot(24)$ together with the solenoidal properties

$$
\begin{aligned}
& \nabla \cdot \mathbf{u}_{2}=0, \\
& \nabla \cdot \mathbf{B}_{2}=0,
\end{aligned}
$$

and Eq. (18) gives

$$
\frac{\partial u_{2 z}}{\partial z}-\frac{U_{0}}{B_{0}} \frac{\partial B_{2 z}}{\partial z}=0
$$

This in turn results in

$$
u_{2 z}=\frac{U_{0}}{B_{0}} B_{2 z}+f(x),
$$

where $f(x)$ can be chosen as zero. We continue by considering Eq. (2) to order $O(E)$,

$$
\begin{aligned}
& \left(\mathbf{u}_{0} \cdot \nabla\right) \mathbf{u}_{2}+\underline{\left(\mathbf{u}_{2} \cdot \nabla\right) \mathbf{u}_{0}}+\left(\mathbf{u}_{1} \cdot \nabla\right) \mathbf{u}_{1} \\
& \quad=-\nabla p_{2}+\left(\mathbf{B}_{0} \cdot \nabla\right) \mathbf{B}_{2}+\underline{\left(\mathbf{B}_{2} \cdot \nabla\right) \mathbf{B}_{0}}+\left(\mathbf{B}_{1} \cdot \nabla\right) \mathbf{B}_{1},
\end{aligned}
$$

where the underlined terms are equal to zero. Writing Eq. (29) on component form and using the $O\left(E^{1 / 2}\right)$ results in Eq. (18) gives

$$
\hat{\mathbf{x}}: \quad U_{0} \frac{\partial u_{2 x}}{\partial z}=-\frac{\partial P_{2}}{\partial x}+B_{0} \frac{\partial B_{2 x}}{\partial z},
$$




$$
\hat{\mathbf{z}}: \quad U_{0} \frac{\partial u_{2 z}}{\partial z}=-\frac{\partial P_{2}}{\partial z}+B_{0} \frac{\partial B_{2 z}}{\partial z},
$$

where

$$
P_{2}=p_{2}+\frac{1}{2} Q^{2}\left(x^{2}+z^{2}\right)-\frac{1}{2}\left(\frac{B_{0} Q}{U_{0}}\right)^{2}\left(x^{2}+z^{2}\right) .
$$

Eliminating the pressure we obtain

$$
U_{0}\left(\frac{\partial^{2} u_{2 x}}{\partial z^{2}}-\frac{\partial^{2} u_{2 z}}{\partial x \partial z}\right)=B_{0}\left(\frac{\partial^{2} B_{2 x}}{\partial z^{2}}-\frac{\partial^{2} B_{2 z}}{\partial x \partial z}\right) .
$$

Equation (28) in Eq. (24) gives together with Eq. (18),

$$
u_{2 x}=\frac{E}{B_{0}}+\frac{U_{0}}{B_{0}} B_{2 x}
$$

Applying Eqs. (28) and (34) in Eq. (33) then results in

$$
\left(\frac{U_{0}^{2}}{B_{0}}-B_{0}\right)\left(\frac{\partial^{2} B_{2 x}}{\partial z^{2}}-\frac{\partial^{2} B_{2 z}}{\partial x \partial z}\right)=0 .
$$

Writing $\mathbf{B}_{2}$ in the vector potential $\mathbf{A}$ such that

$$
\begin{aligned}
& B_{2 x}=-\frac{\partial A}{\partial z}, \\
& B_{2 z}=\frac{\partial A}{\partial x},
\end{aligned}
$$

gives

$$
\frac{\partial^{2} A}{\partial x^{2}}+\frac{\partial^{2} A}{\partial z^{2}}=f(x) .
$$

For $f(x)=0$ we have a rotational-free magnetic field.

\section{COUPLING TO 2D RECONNECTION MODEL}

In this section we continue by coupling the up-to-thispoint obtained results, with the $2 \mathrm{D}$ reconnection model ${ }^{17}$ There the tangential component of the DeHoffmann-Teller ${ }^{22}$ (HT) velocity $U_{t}$ is chosen such that

$$
E+U_{t} B_{n}=0,
$$

where subscript $n$ represents the normal component and $t$ to the tangential component along the Alfvén wave (see Fig. 2). Here $E$ and $B_{n}$ are considered to be of the same order $[O(E)]$, while $U_{t}=O(1)$. From Ref. 17 we have

$$
\begin{aligned}
& U_{t}^{\mathrm{N}}=U_{0}-B_{0}, \\
& U_{t}^{\mathrm{S}}=U_{0}+B_{0} .
\end{aligned}
$$

$\mathrm{N}$ and $\mathrm{S}$ corresponds to the directions north and south of the reconnection site respectively. This gives (cf. Fig. 2)

$$
B_{n}^{\mathrm{N}}=\left.\mathbf{B} \cdot \hat{\mathbf{n}}\right|_{t, n=0}=-\frac{E}{U_{0}-B_{0}}<0,
$$

$$
B_{n}^{S}=\left.\mathbf{B} \cdot \hat{\mathbf{n}}\right|_{t, n=0}=-\frac{E}{U_{0}+B_{0}}>0 .
$$

Figure 2 shows the reconnection structure of the evolution of the rotational discontinuity represented by a large amplitude Alfvén wave, which is followed by a contact discontinuity and a slow shock. Each discontinuity forms an angle with the $z$-axis. In the expressions below we use the angle for the Alfvén wave $\alpha^{\mathrm{N}, \mathrm{S}}$, where $\mathrm{N}$ and $\mathrm{S}$ refers to north and south of the reconnection site, respectively. The unit normal can hence be expressed as

$$
\begin{aligned}
& \hat{\mathbf{n}}=\hat{\mathbf{x}}-\sin \alpha \hat{\mathbf{z}} \approx \hat{\mathbf{x}}-\alpha \hat{\mathbf{z}}, \\
& \left.\mathbf{B} \cdot \hat{\mathbf{n}}\right|_{t, n=0}=B_{x}-\left.\alpha B_{z}\right|_{t, n=0}, \\
& n=x-\alpha z, \\
& t=z+\alpha x .
\end{aligned}
$$

At the boundary represented by the Alfvén wave $n=0$ which corresponds to $x=\alpha z$. This gives

$$
\begin{aligned}
\left.\mathbf{B} \cdot \hat{\mathbf{n}}\right|_{t, n=0}= & B_{x}(\alpha z, z)-\alpha B_{z}(\alpha z, z) \\
= & B_{x}(z, 0)+\frac{\left.\frac{\partial B_{x}}{\partial x}\right|_{0, z} \alpha z-\alpha B_{z}(z, 0)}{} \\
& -\left.\frac{\partial B_{z}}{\partial x}\right|_{0, z} ^{\alpha^{2} z,}
\end{aligned}
$$

where the underlines terms are of order $O\left(E^{2}\right)$, while remaining terms are of order $O(E)$. Recalling that $B_{z}(z, 0)=B_{0}$ according to Eq. (6), we obtain

$$
B_{2 x}(z, 0)=B_{n}+\alpha B_{0} .
$$

Using Eq. (42) we then get

$$
\begin{aligned}
& B_{n}^{\mathrm{N}}=-\frac{E}{U_{0}-B_{0}}+\alpha^{\mathrm{N}} B_{0}, \\
& B_{n}^{\mathrm{S}}=-\frac{E}{U_{0}+B_{0}}-\alpha^{\mathrm{S}} B_{0} .
\end{aligned}
$$

\section{A. Solution for $B_{2}$}

Having $B_{2 x}(z, 0)$ given on the boundary represented by the Alfvén wave (see Fig. 3), we can obtain the general solution using Poisson's formula,

$$
\begin{aligned}
& B_{2 x}(x, z)=\frac{1}{\pi} \int_{-\infty}^{\infty} \frac{x}{x^{2}+\left(z-z^{\prime}\right)^{2}} B_{2 x}\left(x^{\prime}=0, z^{\prime}\right) d z^{\prime}, \\
& B_{2 z}(x, z)=\frac{1}{\pi} \int_{-\infty}^{\infty} \frac{z-z^{\prime}}{x^{2}+\left(z-z^{\prime}\right)^{2}} B_{2 x}\left(x^{\prime}=0, z^{\prime}\right) d z^{\prime},
\end{aligned}
$$

where $B_{2 x}^{\mathrm{N}}\left(x^{\prime}=0, z^{\prime}\right)$ and $B_{2 x}^{\mathrm{S}}\left(x^{\prime}=0, z^{\prime}\right)$ are according to Eq. (50). Equation (50) can then be written as (cf. Fig. 3) 


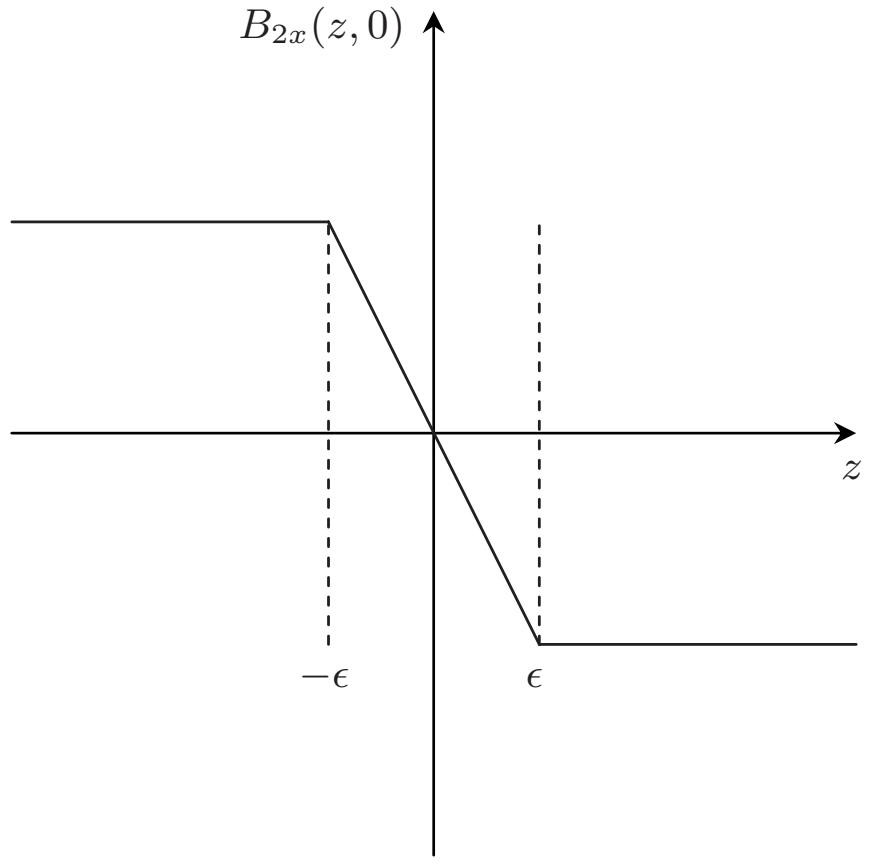

FIG. 3. Variation of $B_{2 x}(z, 0)$.

$$
\begin{aligned}
& B_{n}^{\mathrm{N}}=-\frac{E}{U_{0}+\left|B_{0}\right|}-\alpha^{\mathrm{N}}\left|B_{0}\right|, \quad z>\epsilon, \\
& B_{n}^{\mathrm{S}}=-\frac{E}{U_{0}-\left|B_{0}\right|}+\alpha^{\mathrm{S}}\left|B_{0}\right|, \quad z<\epsilon .
\end{aligned}
$$

\section{Solution for $B_{2 z}$ and the pressure $p_{2}$}

Applying Eq. (54) into Eq. (53) and integrating for $-L$ $<z^{\prime}<-\epsilon$ and $\epsilon<z^{\prime}<L$, respectively, results in

$$
\begin{aligned}
B_{2 z}= & -\frac{1}{2 \pi}\left(\frac{E}{\left|B_{0}\right|-U_{0}}+\alpha^{\mathrm{S}}\left|B_{0}\right|\right) \ln \left[\frac{x^{2}+(z+\epsilon)^{2}}{x^{2}+(z+L)^{2}}\right] \\
& +\frac{1}{2 \pi}\left(\frac{E}{U_{0}+\left|B_{0}\right|}+\alpha^{\mathrm{N}}\left|B_{0}\right|\right) \ln \left[\frac{x^{2}+(z-L)^{2}}{x^{2}+(z-\epsilon)^{2}}\right] .
\end{aligned}
$$

In the limit when the width of the diffusion region approaches zero, i.e., $\epsilon \rightarrow 0$ we then have

$$
\begin{aligned}
B_{2 z}= & -\frac{1}{2 \pi}\left(\frac{E}{\left|B_{0}\right|-U_{0}}+\alpha^{\mathrm{S}}\left|B_{0}\right|\right) \ln \left[\frac{x^{2}+(z+)^{2}}{x^{2}+(z+L)^{2}}\right] \\
& +\frac{1}{2 \pi}\left(\frac{E}{U_{0}+\left|B_{0}\right|}+\alpha^{\mathrm{N}}\left|B_{0}\right|\right) \ln \left[\frac{x^{2}+(z-L)^{2}}{x^{2}+z^{2}}\right] .
\end{aligned}
$$

Considering $L^{2} \gg x^{2}+z^{2}$ we obtain the corresponding result,

$$
B_{2 z}=\frac{1}{2 \pi}\left[\frac{2 E\left|B_{0}\right|}{B_{0}^{2}-U_{0}^{2}}+\left(\alpha^{\mathrm{S}}+\alpha^{\mathrm{N}}\left|B_{0}\right|\right)\right] \ln \left(\frac{L^{2}}{x^{2}+z^{2}}\right) \text {. }
$$

To determine the pressure $p_{2}$ we consider the MHD equation of motion to order $O(E)$ together with the solution for $u_{2 z}$, Eq. (28). This gives $\hat{\mathbf{z}}: \quad\left(\frac{U_{0}^{2}}{B_{0}}-B_{0}\right) \frac{\partial B_{2 z}}{\partial z}=-\frac{\partial}{\partial z}\left[p_{2}+\frac{1}{2} Q^{2} z^{2}-\frac{1}{2}\left(\frac{B_{0}}{U_{0}}\right)^{2} Q^{2} z^{2}\right]$,

$\hat{\mathbf{x}}: \quad\left(\frac{U_{0}^{2}}{B_{0}}-B_{0}\right) \frac{\partial B_{2 x}}{\partial z}=-\frac{\partial}{\partial x}\left[p_{2}+\frac{1}{2} Q^{2} z^{2}-\frac{1}{2}\left(\frac{B_{0}}{U_{0}}\right)^{2} Q^{2} z^{2}\right]$,

which in turn results for the pressure

$$
p_{2}=\frac{1}{2} Q^{2}\left(x^{2}+z^{2}\right)\left[\left(\frac{B_{0}}{U_{0}}\right)^{2}-1\right]+B_{0}\left[1-\left(\frac{U_{0}}{B_{0}}\right)^{2}\right] B_{2 z},
$$

where $B_{2 z}$ is according to Eqs. (56)-(58) above.

\section{Solution for $B_{2 x}$}

We continue to find the complete solution for the ordering $O(E)$ with solving for $B_{2 x}$ in Eq. (52). Applying Eq. (50) in Eq. (52) gives

$$
\begin{aligned}
B_{2 x}(x, z)= & \frac{1}{\pi} \int_{-L}^{-\epsilon} \frac{x}{x^{2}+\left(z-z^{\prime}\right)^{2}}\left(\frac{E}{\left|B_{0}\right|-U_{0}}+\alpha^{\mathrm{S}}\left|B_{0}\right|\right) d z^{\prime} \\
& -\frac{1}{\pi} \int_{\epsilon}^{L} \frac{x}{x^{2}+\left(z-z^{\prime}\right)^{2}}\left(\frac{E}{U_{0}+\left|B_{0}\right|}+\alpha^{\mathrm{N}}\left|B_{0}\right|\right) d z^{\prime},
\end{aligned}
$$

which after performing the integrations result in

$$
\begin{aligned}
B_{2 x}= & \frac{1}{\pi}\left(\frac{E}{\left|B_{0}\right|-U_{0}}+\alpha^{S}\left|B_{0}\right|\right)\left[\arctan \left(\frac{z+L}{x}\right)\right. \\
& \left.-\arctan \left(\frac{z+\epsilon}{x}\right)\right]-\frac{1}{\pi}\left(\frac{E}{U_{0}+\left|B_{0}\right|}+\alpha^{\mathrm{N}}\left|B_{0}\right|\right) \\
& \times\left[\arctan \left(\frac{z-\epsilon}{x}\right)-\arctan \left(\frac{z-L}{x}\right)\right] .
\end{aligned}
$$

For $L \gg z$ and $\epsilon \ll z$ this reduces to

$$
\begin{aligned}
B_{2 x}= & \frac{1}{\pi}\left(\frac{E}{\left|B_{0}\right|-U_{0}}+\alpha^{\mathrm{S}}\left|B_{0}\right|\right)\left[\arctan \left(\frac{L}{x}\right)-\arctan \left(\frac{z}{x}\right)\right] \\
& -\frac{1}{\pi}\left(\frac{E}{U_{0}+\left|B_{0}\right|}+\alpha^{\mathrm{N}}\left|B_{0}\right|\right)\left[\arctan \left(\frac{z}{x}\right)\right. \\
& \left.+\arctan \left(\frac{L}{x}\right)\right] .
\end{aligned}
$$

\section{B. Summary of outer solutions}

We have now obtained an approximate solution for the magnetosheath flow, which can be summarized as follows.

The sought plasma velocity and magnetic field have been expanded about uniform fields $U_{0} \hat{\mathbf{z}}$ and $B_{0} \hat{\mathbf{y}}$, such that [cf. Eq. (3)]

$\mathbf{u}=\mathbf{u}_{0}+\mathbf{u}_{1}+\mathbf{u}_{2}+\cdots$, 


$$
\mathbf{B}=\mathbf{B}_{0}+\mathbf{B}_{1}+\mathbf{B}_{2}+\cdots .
$$

The solution for respective order is

$$
\begin{aligned}
& \mathbf{u}_{0}=U_{0} \hat{\mathbf{z}}, \\
& \mathbf{B}_{0}=B_{0} \hat{\mathbf{z}}, \\
& p_{0}=0, \\
& u_{1 x}=-Q x \text {, } \\
& u_{1 z}=Q z \text {, } \\
& B_{1 x}=-\frac{B_{0}}{U_{0}} Q x, \\
& B_{1 z}=\frac{B_{0}}{U_{0}} Q z, \\
& p_{1}=\left\{\left(\frac{B_{0}}{U_{0}}\right)^{2}-1\right\} U_{0} Q z, \\
& u_{2 x}=\frac{E}{B_{0}}+\frac{U_{0}}{B_{0}} B_{2 x}, \\
& u_{2 z}=\frac{U_{0}}{B_{0}} B_{2 z}, \\
& B_{2 x}=\frac{1}{\pi}\left(\frac{E}{\left|B_{0}\right|-U_{0}}+\alpha^{S}\left|B_{0}\right|\right)\left[\arctan \left(\frac{z+L}{x}\right)\right. \\
& \left.-\arctan \left(\frac{z+\epsilon}{x}\right)\right]-\frac{1}{\pi}\left(\frac{E}{U_{0}+\left|B_{0}\right|}+\alpha^{\mathrm{N}}\left|B_{0}\right|\right) \\
& \times\left[\arctan \left(\frac{z-\epsilon}{x}\right)-\arctan \left(\frac{z-L}{x}\right)\right], \\
& B_{2 z}=-\frac{1}{2 \pi}\left(\frac{E}{\left|B_{0}\right|-U_{0}}+\alpha^{S}\left|B_{0}\right|\right) \ln \left[\frac{x^{2}+(z+\epsilon)^{2}}{x^{2}+(z+L)^{2}}\right] \\
& +\frac{1}{2 \pi}\left(\frac{E}{U_{0}+\left|B_{0}\right|}+\alpha^{\mathrm{N}}\left|B_{0}\right|\right) \ln \left[\frac{x^{2}+(z-L)^{2}}{x^{2}+(z-\epsilon)^{2}}\right], \\
& p_{2}=\frac{1}{2} Q^{2}\left(x^{2}+z^{2}\right)\left[\left(\frac{B_{0}}{U_{0}}\right)^{2}-1\right]+B_{0}\left[1-\left(\frac{U_{0}}{B_{0}}\right)^{2}\right] B_{2 z} \text {. }
\end{aligned}
$$

\section{MATCHING WITH TRANSITION ALFVÉN LAYER SOLUTION}

In this section we match the solutions for the outer plasma flow presented above with the solutions for the tangential component of the velocity and magnetic fields valid in the transition Alfvén layer.

Considering Fig. 2, we see that the tangential vector can be written as

$$
\hat{\mathbf{t}}=\hat{\mathbf{z}}+\alpha \hat{\mathbf{x}}
$$

for small angles $\alpha$. Hence

$$
B_{t}=B_{z}+\alpha B_{x}=B_{0}+B_{1 z}+B_{2 z}+O\left(E^{3 / 2}\right),
$$

considering $\alpha$ to be of order $O(E)$. From the 2D analysis ${ }^{17}$ we have north of the reconnection site,

$$
\begin{aligned}
& B_{t}^{\mathrm{N}}=C_{1} \cdot \operatorname{erf}(\eta)+C_{2}-\frac{U_{\mathrm{HT}}}{2}-\frac{1}{2} \int_{0}^{Z} \frac{1}{U_{\mathrm{HT}}} \frac{\partial P}{\partial Z} d Z, \\
& u_{t}^{\mathrm{N}}=C_{1} \cdot \operatorname{erf}(\eta)+C_{2}+\frac{U_{\mathrm{HT}}}{2}-\frac{1}{2} \int_{0}^{Z} \frac{1}{U_{\mathrm{HT}}} \frac{\partial P}{\partial Z} d Z .
\end{aligned}
$$

Here $\eta$ is the self-similar variable defined as ${ }^{17}$

$$
\eta=\frac{R^{1 / 2} x}{\delta(z)},
$$

where $R$ is the Reynolds number,

$$
R=\frac{R_{E} v_{A}}{\nu},
$$

and $\delta(z)$ the transition layer thickness,

$$
\delta(z)= \pm \frac{2}{U_{\mathrm{HT}}} \sqrt{\int_{0}^{Z} U_{\mathrm{HT}} d \chi} .
$$

Positive sign corresponds to north of the reconnection site, while south of the reconnection site is represented by the negative sign. The total pressure $P$ is

$$
\begin{aligned}
P=p_{1}+p_{2}= & {\left[\left(\frac{B_{0}}{U_{0}}\right)^{2}-1\right]\left[U_{0} Q z+\frac{1}{2} Q^{2}\left(x^{2}+z^{2}\right)\right] } \\
& -\left[1-\left(\frac{U_{0}}{B_{0}}\right)^{2}\right] B_{0} B_{2 z}(x, z) .
\end{aligned}
$$

In the limit when $x \rightarrow 0$ we get

$$
\begin{aligned}
P= & {\left[\left(\frac{B_{0}}{U_{0}}\right)^{2}-1\right]\left[U_{0} Q z+\frac{1}{2} Q^{2} z^{2}\right] } \\
& -\left[1-\left(\frac{U_{0}}{B_{0}}\right)^{2}\right] B_{0} B_{2 z}(z, 0) .
\end{aligned}
$$

\section{A. The HT velocity north and south of the reconnection site}

The HT velocity is expanded in accordance with the velocity and magnetic field such that

$$
U_{\mathrm{HT}}=U_{\mathrm{HT}}^{(0)}+U_{\mathrm{HT}}^{(1)}+U_{\mathrm{HT}}^{(2)}+\cdots,
$$

where $U_{\mathrm{HT}}^{\mathrm{N}(n)}=u_{n z}-B_{n z}, n=0,1,2, \ldots$ north of the reconnection site and $U_{\mathrm{HT}}^{\mathrm{S}(n)}=u_{n z}+B_{n z}, n=0,1,2, \ldots$ south of the reconnection site. Recalling Eq. (67), this results in

$$
\begin{aligned}
& U_{\mathrm{HT}}^{\mathrm{N}(0)}=U_{0}-B_{0}, \\
& U_{\mathrm{HT}}^{\mathrm{N}(1)}=\left(1-\frac{B_{0}}{U_{0}}\right) Q z,
\end{aligned}
$$




$$
\begin{aligned}
& U_{\mathrm{HT}}^{\mathrm{N}(2)}=\left(\frac{U_{0}}{B_{0}}-1\right) B_{2 z}(z, 0), \\
& U_{\mathrm{HT}}^{\mathrm{S}(0)}=U_{0}+B_{0}, \\
& U_{\mathrm{HT}}^{\mathrm{S}(1)}=\left(\frac{B_{0}}{U_{0}}+1\right) Q z, \\
& U_{\mathrm{HT}}^{\mathrm{S}(2)}=\left(\frac{U_{0}}{B_{0}}+1\right) B_{2 z}(z, 0) .
\end{aligned}
$$

\section{B. Maximum reconnection rate}

We may now give an estimate of the maximum reconnection rate in the presence of an outer tangential magnetosheath velocity $U_{0}$. According to Ref. 23 reconnection is shut off when $B_{2 z}$ evaluated close to the diffusion region $(z, x)$ $=(0,0)$ is half the value of the external magnetic field $B_{0}$. From Eq. (78) this condition becomes

$$
B_{2 z}(0,0)=\frac{1}{\pi}\left[\frac{2 E\left|B_{0}\right|}{B_{0}^{2}-U_{0}^{2}}+\left(\alpha^{\mathrm{S}}+\alpha^{\mathrm{N}}\right)\left|B_{0}\right|\right] \ln \left(\frac{L}{\epsilon}\right) \approx \frac{\left|B_{0}\right|}{2} .
$$

To obtain the angles of the Alfvén shocks, it is necessary to solve the complete Riemann reconnection problem including the properties of the magnetosphere (see the Appendix). Inserting these angles of the Alfvén shocks into the condition above gives an estimate of the maximum reconnection rate $E$ as

$$
\begin{aligned}
E_{\max }= & \frac{1}{8}\left[\pi B_{0}\left(4 B_{0} \rho+4 \sqrt{\rho} B_{0}+2 B_{0}-U_{0}+2 \rho U_{0}\right)\right. \\
& \left.\times\left(2 B_{0}+U_{0}\right)\left(B_{0}^{2}-U_{0}^{2}\right)\right] /\left[\left(7 B_{0}^{2} \rho U_{0}+6 B_{0}^{3} \rho\right.\right. \\
& +4 \rho B_{0} U_{0}^{2}+8 B_{0}^{3} \sqrt{\rho}+4 B_{0}^{2} U_{0} \sqrt{\rho} \\
& \left.\left.+2 B_{0}^{3}+\rho U_{0}^{3}\right) \ln \left(\frac{L}{\epsilon}\right)\right],
\end{aligned}
$$

where $\rho$ is the density of the magnetospheric plasma. We note that the reconnection rate goes to zero in the limit $U_{0}$ $\rightarrow B_{0}$, i.e., when the flow becomes Alfvénic, which is a condition we have derived in Ref. 17.

\section{Solutions for the tangential velocity and magnetic field}

Using Eq. (90) the integral terms in Eq. (82) can then be written as

$$
\int_{0}^{Z} \frac{p_{1}^{\prime}+p_{2}^{\prime}}{U_{\mathrm{HT}}^{(0)}+U_{\mathrm{HT}}^{(1)}+U_{\mathrm{HT}}^{(2)}} d z
$$

where $p_{1}^{\prime}=O\left(E^{1 / 2}\right)$ and $p_{2}^{\prime}=O(E)$. This gives

$$
\begin{aligned}
& \int_{0}^{Z} \frac{p_{1}^{\prime}+p_{2}^{\prime}}{U_{\mathrm{HT}}^{(0)}+U_{\mathrm{HT}}^{(1)}+U_{\mathrm{HT}}^{(2)}} d z \\
& \quad=\frac{1}{U_{\mathrm{HT}}^{(0)}} \int_{0}^{Z} p_{1}^{\prime}+p_{2}^{\prime}-p_{1}^{\prime} \frac{U_{\mathrm{HT}}^{(1)}}{U_{\mathrm{HT}}^{(0)}}+O\left(E^{3 / 2}\right) d z \\
& \quad=-\left(1+\frac{B_{0}}{U_{0}}\right) Q z-\left(1+\frac{U_{0}}{B_{0}}\right) B_{2 z}(z, 0), \\
& \left(\frac{B_{0}}{U_{0}}-1\right) Q z+\left(\frac{U_{0}}{B_{0}}-1\right) B_{2 z}(z, 0),
\end{aligned}
$$

north and south of the reconnection site, respectively. This leaves us with the final general expression for the magnetic field,

$$
\begin{aligned}
& B_{t}^{\mathrm{N}}=C_{1} \operatorname{erf}(\eta)+C_{2}-\frac{U_{0}-B_{0}}{2}+\frac{B_{0}}{U_{0}} Q z+B_{2 z}(z, 0), \\
& B_{t}^{\mathrm{S}}=C_{1} \operatorname{erf}(\eta)+C_{2}-\frac{U_{0}+B_{0}}{2}-\frac{B_{0}}{U_{0}} Q z-\frac{U_{0}}{B_{0}} B_{2 z}(z, 0) .
\end{aligned}
$$

To obtain a complete solution, we consider a matching when $\eta \rightarrow \pm \infty$ such that

$$
\begin{aligned}
& \eta \rightarrow+\infty: \quad B_{t} \rightarrow B_{0 z}+B_{1 z}+B_{2 z}=B_{0}+B_{1 z}+B_{2 z}(z, 0), \\
& \eta \rightarrow-\infty: \quad B_{t} \rightarrow-B_{0}+B_{1 z}+B_{2 z}(z, 0),
\end{aligned}
$$

which results in the tangential component of the magnetic field valid north and south of the reconnection site,

$$
B_{t}=B_{0} \operatorname{erf}(\eta)+\frac{B_{0}}{U_{0}} Q z+B_{2 z}(z, 0) .
$$

North of the reconnection site $u_{t}=B_{t}+U_{\mathrm{HT}}$. With $B_{t}$ and the HT velocity defined above, we obtain

$$
u_{t}^{\mathrm{N}}=B_{0} \operatorname{erf}(\eta)+U_{0}-B_{0}+Q z+\frac{U_{0}}{B_{0}} B_{2 z}(z, 0) .
$$

South of the reconnection site $u_{t}=U_{\mathrm{HT}}-B_{t}$. With the HT velocity according to Eq. (90) we get the corresponding solution,

$$
u_{t}^{\mathrm{S}}=-B_{0} \operatorname{erf}(\eta)+U_{0}+B_{0}+\frac{U_{0}}{B_{0}} B_{2 z}(z, 0)+Q z .
$$

Now we have the outer solution (o) for the magnetosheath flow and the inner (i) solution for the flow in the transition Alfvén layer. In order to obtain a solution valid in both regions we construct a composite solution (c) such that ${ }^{24}$

$$
\mathbf{u}^{\mathrm{c}}=\mathbf{u}^{\mathrm{o}}+\mathbf{u}^{\mathrm{i}}-\mathbf{u}^{\mathrm{o}^{\mathrm{i}}},
$$

where the last term is the inner limit of the outer solution, which in turn is identical to the outer limit of the inner solution. The composite solution for the magnetic field is constructed analogously. 


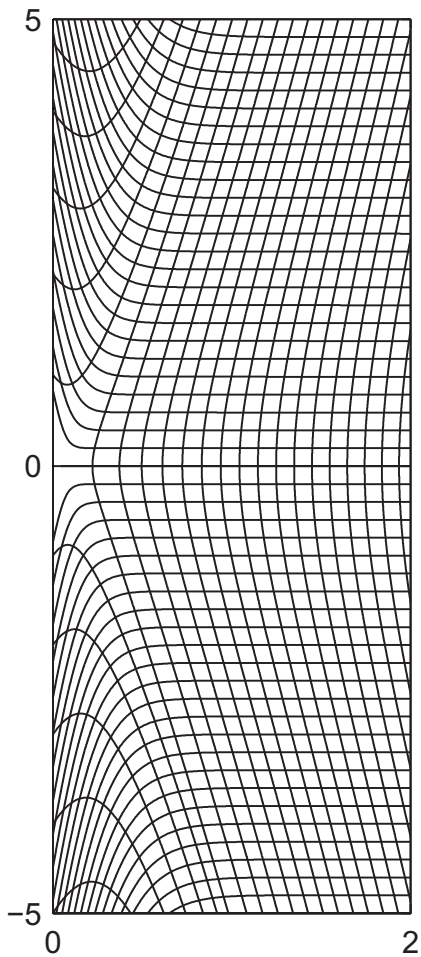

(a) $R=100$

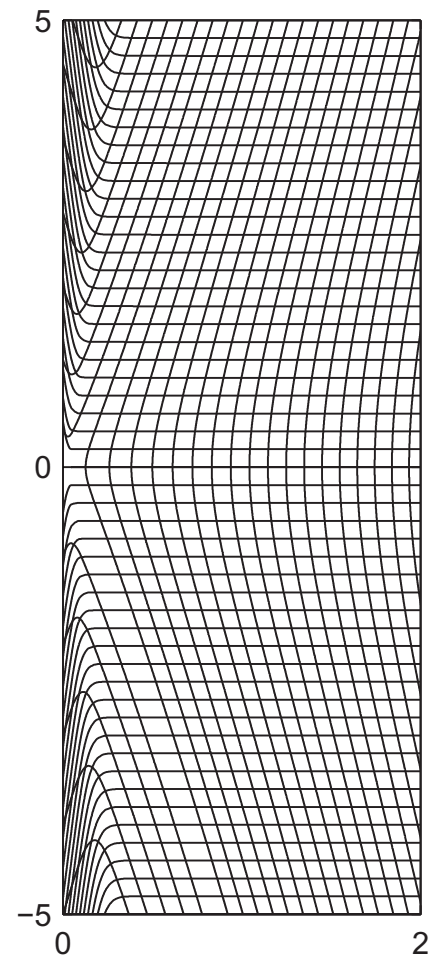

(b) $R=2500$

FIG. 4. Magnetic field lines and streamlines for the ideal case, where $U_{0}$ $=0$ and $Q=0$ with a Reynolds number of (a) $R=100$ and (b) $R=2500$. The angles for both (a) and (b) are $\alpha^{\mathrm{N}}=\alpha^{\mathrm{S}}=11^{\circ}$. Both the field lines and the streamlines are solid curves. Horizontal axis: $x$; vertical axis: $z$.

\section{RESULTS: STREAMLINE AND MAGNETIC FIELD DEVELOPMENT}

In Fig. 4, two examples of the Petschek-like solution are presented with two different Reynolds numbers. The higher Reynolds number $(R=2500)$ produces a sharper transition through the Alfvén layer and emphasizes how the streamlines behave, and is therefore used in subsequent plots. The influence of the velocity gradient $Q$ manifests itself mainly by how the transition layer south of the reconnection site gets thicker with an increasing value of the gradient. The thickness of the transition layer north of the reconnection site does not change much, but the magnetic field strength in the layer does get weaker with increasing $Q$, see Fig. 5. When the value of the gradient gets high enough, some of the magnetic field lines coming from the north side are diverted into the magnetopause, leaving a portion of the area outside the reconnection site without any field lines. Also, Figs. 4-6 show that the streamlines become dramatically distorted. The distortion on the north side has its origin in the Petschek-like case where the plasma stream diverges at the reconnection site, see Figs. 5 and 6. This divergence is pushed northward and this gives rise to the distorted topology of the streamlines. When looking at the effects of the uniform velocity $U_{0}$, it can be seen that the effect of a higher value of $U_{0}$ is a decrease in thickness of the transition layer south of the reconnection site. This is the opposite effect of what was seen with increasing $Q$. On the north side, Fig. 6 shows that the transition layer gets slightly thicker as $U_{0}$ increases.

\section{SUMMARY}

In this study we consider the stationary MHD Riemann problem including dissipation in the form of resistivity and viscosity for the intermediate shock wave at the terrestrial magnetopause. It is explicitly shown how dissipation affects the thickness of this wave in the vicinity of a reconnection site at the dayside magnetopause boundary. Tangential velocity shear across the complete reconnection structure from the magnetosheath side into the magnetosphere is included as well as nonzero velocity gradients.

The solution combines a 2D viscous/resistive reconnection model by Westerberg and Åkersted ${ }^{17}$ of the plasma flow through the Alfvén transition layer, with solutions for the outer magnetosheath flow based on the Petschek ${ }^{23}$ solution methodology in his classical model treating fast magnetic reconnection. Thereby, the $2 \mathrm{D}$ model is put into the context of a well established reconnection model as the influence of the reconnection process on the magnetosheath flow is investigated.

The ideal MHD equations governing the outer magnetosheath flow are solved approximately using an expansion in orders of the electric field, along uniform velocity and magnetic fields. Considering solutions up to the order $O(E)$ for the outer magnetosheath flow, we obtain a class of solutions which are matched with the 2D viscous/resistive solutions in the transition Alfvén layer. It is shown that a Petschek-like solution is obtained when the velocity gradient and the uniform velocity are zero. Having nonzero values on these parameters dramatically changes the topology of the magnetic field line and plasma streamline pattern. The reconnection rate is also modified substantially, leading to a shut off when the velocity reaches the Alfvén velocity.

\section{ACKNOWLEDGMENTS}

This project was partly financed by the Swedish National Graduate School of Space Technology.

\section{APPENDIX: ANGLES OF IDEAL INCOMPRESSIBLE MHD SHOCK WAVES}

Here we present results regarding the angles of the different shock waves in the ideal incompressible MHD limit. A is the Alfven wave, $S$ is the incompressible limit of a slow shock, and $C$ is the contact discontinuity. The angles can be calculated from the usual jump conditions across the different waves. ${ }^{25}$ To obtain the angles it is necessary to solve the complete reconnection problem including the influence from the magnetospheric side. See Fig. 2. Results regarding the angles have been given by Ref. 26 for the true compressible ideal MHD case but with the restriction of no tangential flow. The novelty here is that we present results for the case with a finite tangential magnetosheath velocity with shear and gradient. The analysis is quite complicated and only the results will be presented. Respective angle is hence as follows: 


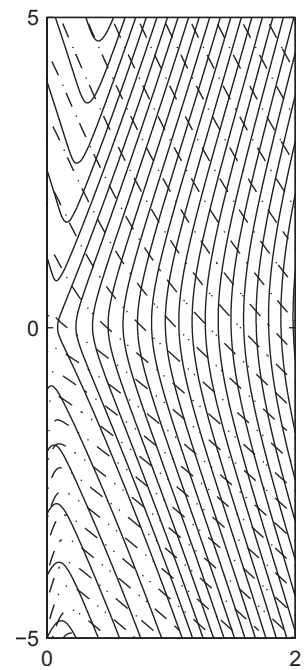

(a) $Q=0$

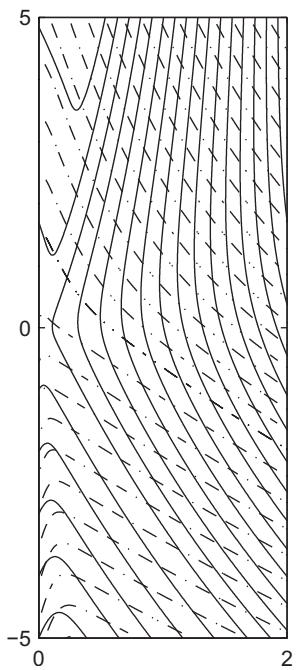

(b) $Q=0.02$

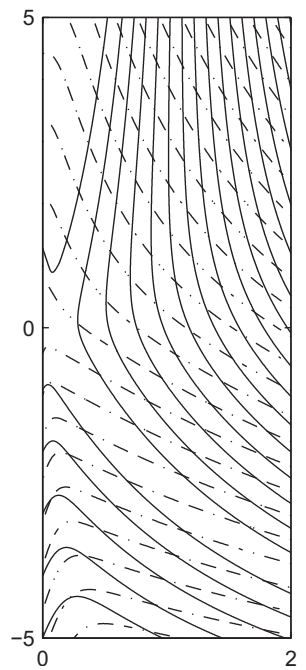

(c) $Q=0.04$

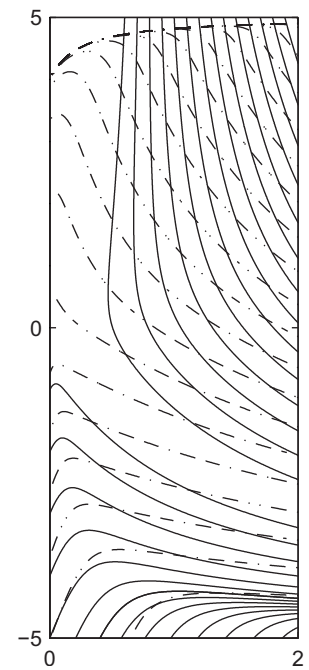

(d) $Q=0.06$

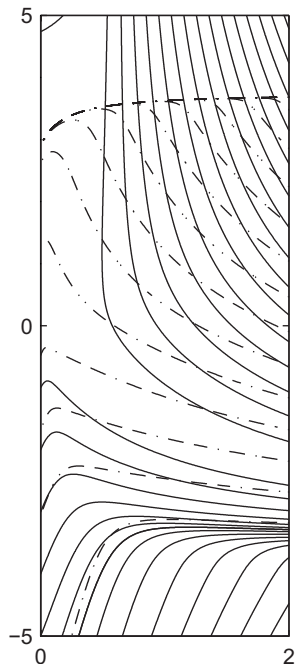

(e) $Q=0.08$

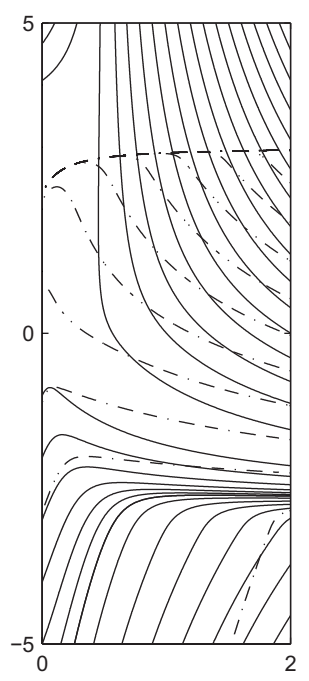

(f) $Q=0.10$

FIG. 5. Magnetic field lines (solid lines) and streamlines (dash dotted lines) for different values of $Q$ with constant $U_{0}=0.30$. The density of the magnetosphere plasma is $\rho=0.05$. The angles $\alpha^{\mathrm{N}}$ and $\alpha^{\mathrm{S}}$ are constant at $25^{\circ}$ and $6.6^{\circ}$, respectively. Horizontal axis: $x$; vertical axis: $z$.

$$
\begin{aligned}
\alpha^{\mathrm{N}}= & E\left(4 B_{0}^{3} \rho-2 B_{0} U_{0}^{2} \rho-4 U_{0}^{2} B_{0} \sqrt{\rho}-4 B_{0}^{2} U_{0} \sqrt{\rho}+8 B_{0}^{3} \sqrt{\rho}-4 U_{0} B_{0}^{2}-2 B_{0}^{2} U_{0} \rho+U_{0}^{3}+U_{0}^{2} B_{0}\right) /\left[\left(2 B_{0}+U_{0}\right)\left(B_{0}-U_{0}\right)\right. \\
& \left.\times\left(4 B_{0} \rho+4 B_{0} \sqrt{\rho}+2 B_{0}-U_{0}+2 U_{0} \rho\right)\left(U_{0}+B_{0}\right) B_{0}\right],
\end{aligned}
$$

$$
\begin{aligned}
\alpha^{\mathrm{S}}= & E\left(4 B_{0}^{3} \rho+4 U_{0}^{3} \rho+14 B_{0} U_{0}^{2} \rho+14 B_{0}^{2} U_{0} \rho+8 B_{0}^{3} \sqrt{\rho}+4 U_{0}^{2} B_{0} \sqrt{\rho}-12 B_{0}^{2} U_{0} \rho-U_{0}^{3}+U_{0}^{2} B_{0}+4 U_{0} B_{0}^{2}\right) /\left[\left(2 B_{0}+U_{0}\right)\left(B_{0}-U_{0}\right)\right. \\
& \left.\times\left(4 B_{0} \rho+4 B_{0} \sqrt{\rho}+2 B_{0}-U_{0}+2 U_{0} \rho\right)\left(U_{0}+B_{0}\right) B_{0}\right],
\end{aligned}
$$

$$
\begin{aligned}
\widetilde{\alpha}= & -E\left(2 U_{0}^{2} B_{0}^{2}+U_{0}^{4} \rho-2 U_{0}^{3} B_{0} \rho^{3 / 2}+2 B_{0}^{3} U_{0} \rho^{3 / 2}-4 B_{0}^{2} U_{0}^{2} \rho^{3 / 2}+4 B_{0}^{4} \rho^{3 / 2}-4 B_{0}^{4}+U_{0}^{2} B_{0}^{2} \sqrt{\rho}+U_{0}^{4} \sqrt{\rho}-8 B_{0}^{4} \sqrt{\rho}-5 U_{0}^{2} B_{0}^{2} \rho\right. \\
& \left.+2 U_{0}^{3} B_{0} \sqrt{\rho}-4 B_{0}^{3} U_{0} \sqrt{\rho}-2 B_{0}^{3} U_{0} \rho\right) /\left[( 2 B _ { 0 } \sqrt { \rho } + U _ { 0 } \sqrt { \rho } + B _ { 0 } ) B _ { 0 } ( B _ { 0 } - U _ { 0 } ) ( 2 B _ { 0 } + U _ { 0 } ) ( U _ { 0 } + B _ { 0 } ) \left(6 B_{0} \sqrt{\rho}+2 B_{0}+4 B_{0} \rho^{3 / 2}\right.\right. \\
& \left.\left.+8 B_{0} \rho+2 U_{0} \rho^{3 / 2}+2 U_{0} \rho-U_{0} \rho-U_{0}\right)\right],
\end{aligned}
$$

$$
\begin{aligned}
\alpha^{\mathrm{CN}}= & -E\left(4 B_{0}^{3} \rho-2 B_{0}^{3}+2 B_{0}^{3} \sqrt{\rho}-U_{0} B_{0}^{2}-B_{0}^{2} U_{0} \sqrt{\rho}-4 B_{0} U_{0} \rho-4 U_{0}^{2} B_{0} \sqrt{\rho}+U_{0}^{3}+U_{0}^{3} \sqrt{\rho}\right) /\left[(1+\sqrt{\rho})\left(2 B_{0}+U_{0}\right)\left(B_{0}-U_{0}\right)\right. \\
& \left.\times\left(4 B_{0} \sqrt{\rho}+4 B_{0} \rho+2 B_{0}-U_{0}+2 \rho U_{0}\right)\left(U_{0}+B_{0}\right) B_{0}\right],
\end{aligned}
$$




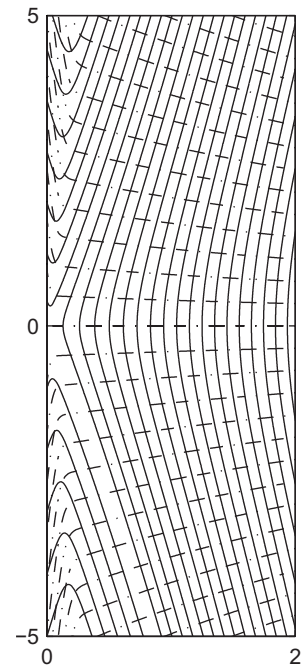

(a) $U_{0}=0$ $\alpha^{\mathrm{N}}=\alpha^{\mathrm{S}}=11^{\circ}$

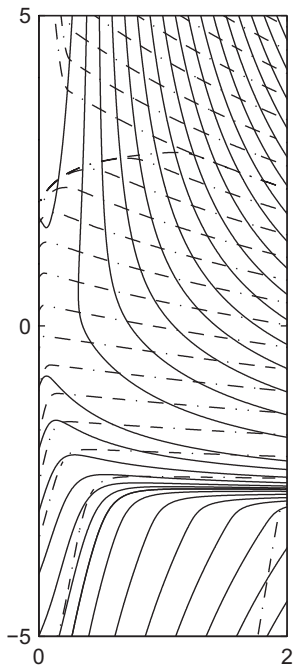

(b) $U_{0}=0.06$; $\alpha^{\mathrm{N}}=14^{\circ} ; \alpha^{\mathrm{S}}=10^{\circ}$
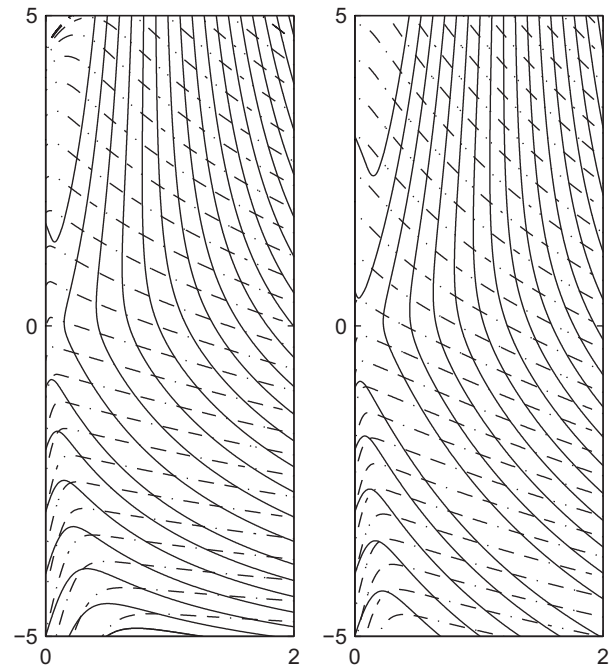

(c) $U_{0}=0.12 ; \quad(\mathrm{d}) U_{0}=0.18$;

$\alpha^{\mathrm{N}}=16^{\circ} ; \alpha^{\mathrm{S}}=8.9^{\circ}$

$\alpha^{\mathrm{N}}=19^{\circ} ; \alpha^{\mathrm{S}}=8.0^{\circ}$

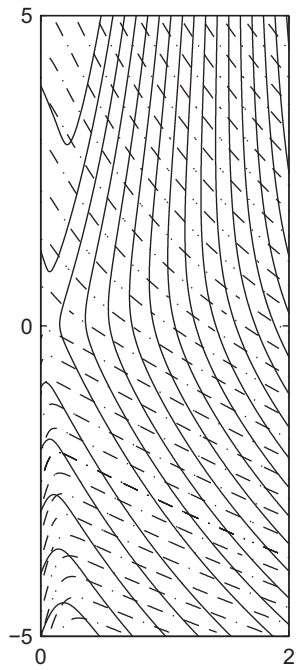

(e) $U_{0}=0.24$

$\alpha^{\mathrm{N}}=22^{\circ} ; \alpha^{\mathrm{S}}=7.2^{\circ}$

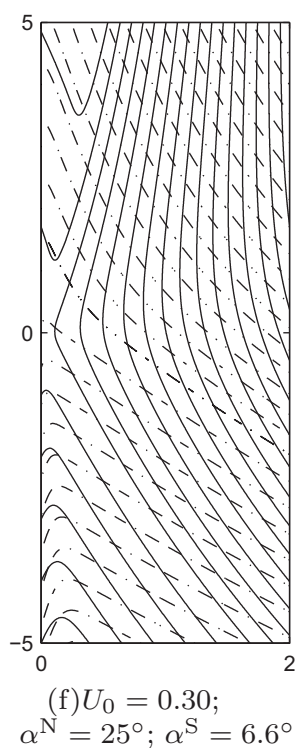

FIG. 6. Magnetic field lines (solid lines) and streamlines (dash dotted lines) for different values of $U_{0}$ with constant $Q=0.02$. The density of the magnetosphere plasma is $\rho=0.05$. Horizontal axis: $x$; vertical axis: $z$.

$$
\begin{aligned}
\alpha^{\mathrm{CS}}= & -E\left(8 B_{0}^{3} U_{0} \rho-8 B_{0} U_{0}^{3} \rho-4 U_{0}^{4} \rho+4 U_{0}^{2} B_{0}^{2} \rho+8 B_{0}^{4} \sqrt{\rho}+4 B_{0}^{3} U_{0} \sqrt{\rho}-8 U_{0}^{2} B_{0}^{2} \sqrt{\rho}-4 U_{0}^{3} B_{0} \sqrt{\rho}-U_{0}^{2} B_{0}^{2}-2 U_{0}^{3} B_{0}+4 U_{0} B_{0}^{3}+U_{0}^{4}\right. \\
& \left.-4 B_{0}^{4}\right) /\left[\left(2 B_{0}+U_{0}\right)\left(B_{0}-U_{0}\right)\left(4 B_{0} \rho+4 B_{0} \sqrt{\rho}+2 B_{0}-U_{0}+2 \rho U_{0}\right)\left(U_{0}+B_{0}\right)\left(2 B_{0}-U_{0}\right) B_{0}\right] .
\end{aligned}
$$

Here $\rho$ is the density of the magnetospheric plasma.

\footnotetext{
${ }^{1}$ A. Vaivads, Y. Khotyaintsev, M. André, A. Retinò, S. C. Buchert, B. N. Rogers, P. Décréau, G. Paschmann, and T. D. Phan, Phys. Rev. Lett. 93, 105001 (2004).

${ }^{2}$ M. André, A. Vaivads, S. C. Buchert, A. N. Fazakerley, and A. Lahiff, Geophys. Res. Lett. 31, L03803, DOI:10.1029/2003GL018137 (2004).

${ }^{3}$ A. Retinò, M. B. B. Cattaneo, M. F. Marcucci, A. Vaivads, M. André, Y. Khotyaintsev, T. Phan, G. Pallocchia, H. Reme, E. Möbius, B. Klecker, C. W. Carlson, M. McCarthy, A. Korth, R. Lundin, and A. Balogh, Ann. Geophys. 23, 461 (2005).

${ }^{4}$ A. Retinò, A. Vaivads, M. André, F. Sahraoui, Y. Khotyaintsev, J. S. Pickett, M. B. B. Cattaneo, M. F. Marcucci, M. Morooka, C. J. Owen, S.
}

C. Buchert, and N. Cornilleau-Wehrlin, Geophys. Res. Lett. 33, L06101, DOI: 10.1029/2005GL024650 (2006).

${ }^{5}$ M. Palmroth, P. Janhunen, T. I. Pulkkinen, and H. E. J. Koskinen, Ann. Geophys. 22, 549 (2004).

${ }^{6}$ M. Palmroth, T. V. Laitinen, and T. I. Pulkkinen, Ann. Geophys. 24, 3467 (2006).

${ }^{7}$ T. V. Laitinen, P. Janhunen, T. I. Pulkkinen, M. Palmroth, and H. E. J. Koskinen, Ann. Geophys. 24, 3059 (2006).

${ }^{8}$ M. Ugai, Phys. Plasmas 14, 062902 (2007).

${ }^{9}$ M. Ugai, Phys. Plasmas 15, 082306 (2008).

${ }^{10}$ M. Ugai, Phys. Plasmas 16, 012901 (2009).

${ }^{11}$ Y. Lin, L. C. Lee, and C. F. Kennel, Geophys. Res. Lett. 19, 229, 
DOI:10.1029/91GL03008 (1992).

${ }^{12}$ Y. Lin and L. C. Lee, Geophys. Res. Lett. 21, 855, DOI:10.1029/ 94GL00704 (1994).

${ }^{13}$ Y. Lin and L. C. Lee, Space Sci. Rev. 65, 59 (1994).

${ }^{14}$ Y. Lin and H. Xie, Geophys. Res. Lett. 24, 3145, DOI:10.1029/ 97GL03278 (1997).

${ }^{15}$ Y. Lin and L. C. Lee, Phys. Plasmas 6, 3131 (1999).

${ }^{16}$ X. Sun, Y. Lin, and X. Wang, Phys. Plasmas 12, 012305 (2005).

${ }^{17}$ L. G. Westerberg and H. O. Åkerstedt, J. Plasma Phys. 71, 537 (2005).

${ }^{18}$ L. G. Westerberg and H. O. Åkerstedt, J. Plasma Phys. 72, 359 (2006).

${ }^{19}$ L. G. Westerberg and H. O. Åkerstedt, J. Plasma Phys. 73, 89 (2007).

${ }^{20}$ L. G. Westerberg, H. O. Åkerstedt, H. Nilsson, H. Rème, and A. Balogh, J. Geophys. Res. 113, A03205, DOI:10.1029/2007JA012546 (2008).
${ }^{21}$ N. E. Erkaev, V. S. Semenov, and F. Jamitzky, Phys. Rev. Lett. 84, 1455 (2000).

${ }^{22}$ F. DeHoffmann and E. Teller, Phys. Rev. Lett. 80, 692 (1950).

${ }^{23} \mathrm{H}$. Petschek, in Magnetic Field Annihilation, Proceedings of the AASNASA Symposium, Goddard Space Flight Center, Greenbelt, MD, October 28-30, 1963, edited by W. N. Hess (1964), Vol. 50, p. 425.

${ }^{24}$ A. H. Nayfeh, Introduction to Perturbation Techniques (Wiley, New York, 1993).

${ }^{25}$ T. J. M. Boyd and J. J. Sanderson, Plasma Dynamics (Nelson, London, 1969).

${ }^{26}$ M. F. Heyn, H. K. Biernat, V. S. Semenov, and I. V. Kubyshkin, J. Geophys. Res. 90, 1781, DOI:10.1029/JA090iA02p01781 (1985). 